\title{
Morbilidad y mortalidad de la linfadenectomía cervical. Análisis de 311 casos
}

\author{
Morbidity and mortality of cervical lymphenectomy. Analysis of 311 cases
}

Héctor Hernández-Vargas ${ }^{1}$, Edgar J. Cortes-Torres ${ }^{1}$, Alejandro González-Ojeda², Jacob E. Pérez-Landeros², Francisco J. Barrera-López ${ }^{2}$ y Clotilde Fuentes-Orozco ${ }^{2 *}$

${ }^{1}$ Servicio de Cirugía Oncológica; ${ }^{2}$ Unidad de Investigación Biomédica. Unidad Médica de Alta Especialidad, Hospital de Especialidades del Centro Médico Nacional de Occidente, Instituto Mexicano del Seguro Social, Guadalajara, Jalisco, México

\section{Resumen}

Objetivo: Determinar la morbimortalidad y los factores de riesgo asociados a la linfadenectomía cervical. Métodos: Estudio transversal analítico en el que se incluyeron pacientes sometidos a linfadenectomía cervical en cualquiera de sus variantes, secundarios a la recurrencia de alguna enfermedad neoplásica, desde enero de 2011 hasta noviembre de 2016. Resultados: Se incluyeron 311 pacientes, de los cuales 203 (65.3\%) eran mujeres, con una edad de $49 \pm 17.2$ años. Los diagnósticos más frecuentes fueron cáncer de tiroides, en 194 (62.4\%) casos, y cáncer de laringe recurrente, en 22 (7.1\%). Cursaron asintomáticos 166 (53.4\%) pacientes, y 119 (38.3\%) presentaron masas o nódulos en el cuello, siendo el nivel III el más afectado, con 276 (88.7\%) pacientes. La morbilidad general fue del 17\% y la mortalidad fue del 5.8\%. Los factores relacionados con la mortalidad fueron la sintomatología presente $(p=0.006)$, la afectación del nivel l $(p=0.005)$, la disección ganglionar extensa $(p=0.01)$ y las complicaciones vasculares $(p=0.0001)$ y de la herida $(p=0.01)$. Conclusiones: Debido al patrón de diseminación linfática de los tumores de cabeza y cuello, la linfadenectomía selectiva desempeña un papel crucial en su tratamiento. Se ha optado por la realización de cirugías más conservadoras, siempre y cuando sean preservados los principales objetivos oncológicos.

PALABRAS CLAVE: Linfadenectomía cervical. Niveles ganglionares. Morbilidad. Mortalidad.

\begin{abstract}
Objective: To determine the morbidity and mortality and the risk factors associated with cervical lymphadenectomy. Method: Cross-sectional study; patients undergoing cervical lymphadenectomy were included in any of its variants, secondary to the recurrence of some neoplastic entity; from January 2011 to November 2016. Results: 311 patients were included of which $65.3 \%$ were women and $49 \pm 17.2$ years old. The most frequent diagnoses were thyroid cancer 194 (62.4\%) and recurrent laryngeal cancer 22 (7.1\%). Asymptomatic patients had 53.4\% of the patients and 119 (38.3\%) presented masses or nodules in the neck, with level III being the most affected with 276 (88.7\%) patients. Overall morbidity was $17 \%$ and mortality was $5.8 \%$. The factors related to mortality were: present symptomatology $(p=0.006)$, involvement of level I $(p=0.005)$, extensive lymph node dissection $(p=0.01)$, and vascular complications $(p=0.0001)$ and the wound $(p=0.01)$. Conclusions: Due to the lymphatic dissemination pattern of head and neck tumors, selective lymphadenectomy plays a crucial role in the treatment of these neoplasms. We have opted to perform more conservative surgeries, as long as the main oncological objectives are preserved.
\end{abstract}

KEY WORDS: Cervical lymphadenectomy. Ganglion levels. Morbidity. Mortality.

\author{
Correspondencia: \\ ${ }^{*}$ Clotilde Fuentes Orozco \\ Belisario Domínguez, 1000 \\ Col. Independencia Oblatos \\ C.P. 44349 , Guadalajara, Jal., México \\ E-mail: clotilde.fuentes@ gmail.com
}

Fecha de recepción: 27-03-2018

Fecha de aceptación: 05-06-2018

DOI: 10.24875/CIRU.18000311
Cir Cir. 2018;86:522-527

Contents available at PubMed www.cirugiaycirujanos.com 


\section{Introducción}

Se ha estimado que la presencia de metástasis linfáticas indica una disminución del $50 \%$ en la supervivencia global en pacientes con cualquier entidad nosológica, y la afectación ganglionar contralateral indica otra disminución del 50\%. Por lo tanto, es importante realizar la disección ganglionar cervical, ya que contribuye a la estadificación de ciertos tumores y es considerada como un factor pronóstico ${ }^{1-3}$.

La disección del cuello en sus diversas formas es el procedimiento estándar para el tratamiento quirúrgico de las metástasis de ganglios linfáticos cervicales, pues permite la limpieza extensa de todos los ganglios linfáticos con crecimiento macroscópico y ofrece una información histológica exacta sobre los ganglios con riesgo de presentar micrometástasis en el cuello clínicamente negativo, cuyo objetivo es el control local de la enfermedad ${ }^{4}$. Las opiniones varían en cuanto a las indicaciones para la disección del cuello y el tipo de disección para diferentes situaciones ${ }^{5,6}$.

Actualmente se clasifica la linfadenectomía cervical en cuatro categorías: disección radical del cuello, disección radical del cuello modificada, disección selectiva del cuello y disección del cuello extendida. La transición de disección radical a disección selectiva del cuello se ha traducido en un menor número de complicaciones y una menor morbilidad, al mismo tiempo que se preservan la eficacia quirúrgica y el cumplimiento de los principios oncológicos ${ }^{7,8}$.

Las complicaciones operatorias, como lesión de vasos o nervios, son causa de morbilidad mayor, esto aunado a las complicaciones médicas no relacionadas con el procedimiento que pueden presentarse después de la linfadenectomía cervical. Aunque poco frecuente, la muerte puede sobrevenir como consecuencia de morbilidad grave ${ }^{9,10}$.

El propósito de este estudio fue evaluar las complicaciones y la mortalidad asociadas al procedimiento de linfadenectomía cervical, ya que en nuestra institución constituye uno de los principales motivos de consulta, así como de procedimientos quirúrgicos, por lo que es necesario detectar los diversos factores de riesgo asociados a la morbimortalidad con el propósito de implementar mejoras que disminuyan su frecuencia.

\section{Método}

Se realizó un estudio transversal analítico. Se revisó la base de datos del departamento de cirugía oncológica y se incluyeron todos los pacientes a quienes se realizó linfadenectomía cervical en cualquiera de sus variantes como procedimiento quirúrgico, debido a recurrencia ganglionar de alguna neoplasia durante el periodo del 1 de enero de 2011 hasta el 30 de noviembre de 2016. Se excluyeron los pacientes que contaran con criterios de irresecabilidad, tales como afección de columna vertebral o médula espinal, y aquellos con comorbilidad grave que impidiera dicho procedimiento.

Se recabaron variables como edad, sexo, síntomas de presentación, neoplasia primaria y tipo de cirugía realizada (disección radical, modificada, selectiva, suprahomoiodea, lateral, posterolateral, central y posterior), y se compararon con el resultado de histopatología, las complicaciones posquirúrgicas asociadas al procedimiento (propias de la herida, vasculares, nerviosas y fístula quilosa) y la mortalidad posquirúrgica.

\section{Análisis estadístico}

Las variables fueron capturadas en una base de datos electrónica y se sometieron a un análisis estadístico utilizando el programa SPSS (versión 21.0; IBM Corp., Armonk, NY, EE.UU.). Se obtuvieron números crudos y porcentajes para las variables de tipo cualitativo, y medias con su desviación estándar para las variables de tipo cuantitativo. La fase inferencial se hizo mediante un análisis univariado con prueba de ji al cuadrado o exacta de Fisher para variables cualitativas y cálculo de razones de momios (OR) e intervalos de confianza (IC) del 95\%. Todo valor de $p<0.05$ se consideró estadísticamente significativo.

\section{Consideraciones éticas}

Los aspectos éticos en este estudio se basan en el Reglamento de la Ley General de Salud en Materia de Investigación para la Salud y en la Declaración de Helsinki y sus enmiendas, así como en los códigos nacionales e internacionales vigentes para las buenas prácticas en la investigación. Fue autorizado con el número de registro del Comité Local de Investigación y Ética en Investigación en Salud R-2016-1301-117. Se realizó con recursos propios de la institución, por lo que se declara que no existe conflicto de intereses.

\section{Resultados}

Se incluyeron 311 pacientes, 108 (34.7\%) de sexo masculino y $203(65.3 \%)$ de sexo femenino. La edad fue de $49 \pm 17.2$ años. El diagnóstico más frecuente fue cáncer de tiroides, en 194 pacientes (62.4\%), 
seguido de cáncer de laringe recurrente en 22 (7.1\%) pacientes, y de metástasis cervical de primario desconocido en 16 (5.1\%) pacientes. En relación con los síntomas, $166(53.4 \%)$ pacientes cursaron asintomáticos y 119 (38.3\%) refirieron la presencia de masas o nódulos en el cuello; de ellos, 26 (8.4\%) tenían síntomas tales como disfagia o disfonía.

En cuanto a la disección quirúrgica, 108 (34.7\%) se sometieron a disección ganglionar lateral, $78(25 \%)$ a disección radical modificada tipo 3 y 48 (15.4\%) a disección supraomohioidea (Tabla 1). Del total de los ganglios resecados, $47(15.1 \%)$ fueron benignos y 264 (84.9\%) fueron positivos a malignidad.

Los niveles ganglionares resecados correspondieron en 185 pacientes (59.4\%) al nivel III, en 177 (56.9\%) al nivel II, en 100 (32.1\%) al nivel IV, en 66 (21.2\%) al nivel I, en $45(14.4 \%)$ al nivel $\mathrm{VI}$, en $28(9 \%)$ al nivel V, y en $16(5.1 \%)$ al nivel VII.

\section{Complicaciones vasculares}

Se analizaron los factores de riesgo asociados a complicaciones vasculares, de las cuales se encontraron hipertensión arterial sistémica (OR: 2.7; IC 95\%: 1.1-6.5; $p=0.02$ ), síntomas clínicos (OR: 2.8; IC 95\%: 1.1-7.1; $\mathrm{p}=0.01$ ), disección radical tipo III (OR: 3; IC 95\%: 1.2$7.1 ; p=0.01$ ) y disección suprahomoioidea (OR: 0.9; IC $95 \% 0.8-0.9 ; p=0.01$ ), como se observa en la tabla 2 .

\section{Complicaciones nerviosas}

Se encontraron lesiones del nervio hipogloso mayor en $9(2.8 \%)$ pacientes y del nervio laríngeo recurrente en $1(0.3 \%)$, las cuales se asociaron con resección de nivel I (OR: 19.1; IC 95\%: 4.0-91.2; $p=0.00$ ) y de nivel II (OR: 1; IC 95\%: 1.0-1.1; $p=0.002$ ).

\section{Complicaciones de la herida}

Las complicaciones de la herida quirúrgica fueron infección en $3(0.9 \%)$ pacientes, dehiscencia de la herida quirúrgica en $8(2.5 \%)$ y seroma en $2(0.6 \%)$. Se observó un mayor riesgo en pacientes de sexo masculino (OR: 0.2; IC 95\%: 0.1-0.7; $p=0.009$ ), con diabetes mellitus (OR: 2.8; IC 95\%: 1.0-7.5; $p=0.04$ ) y con disección lateral (OR: 0.3; IC 95\%: 0.09-1.1; $p=0.05$ ).

\section{Fístula quilosa}

En los 12 (3.85\%) pacientes que desarrollaron fístula quilosa se encontraron como factores de riesgo
Tabla 1. Características demográficas y clínicas de la población de estudio

\begin{tabular}{|c|c|}
\hline Variable & n (\%) \\
\hline \multicolumn{2}{|l|}{ Sexo } \\
\hline Masculino & $108(34.7)$ \\
\hline Femenino & $203(65.3)$ \\
\hline Edad (años) & $49 \pm 17.2$ \\
\hline Estancia hospitalaria (días) & $4.2 \pm 4$ \\
\hline \multicolumn{2}{|l|}{ Diagnóstico } \\
\hline Cáncer de tiroides & $194(62.4)$ \\
\hline Cáncer de laringe & $22(7.1)$ \\
\hline Metástasis cervical de primario desconocido & $16(5.1)$ \\
\hline Cáncer de parótida & $11(3.5)$ \\
\hline Melanoma & $11(3.5)$ \\
\hline Cáncer de lengua & $9(2.9)$ \\
\hline Otros & $48(15.6)$ \\
\hline \multicolumn{2}{|l|}{ Cuadro clínico } \\
\hline Asintomático & $166(53.4)$ \\
\hline Masas o nódulos & $119(38.3)$ \\
\hline Otros & $26(8.4)$ \\
\hline \multicolumn{2}{|l|}{ Morbilidad } \\
\hline Complicaciones vasculares & $23(7.4)$ \\
\hline Complicaciones nerviosas & $11(3.5)$ \\
\hline Complicaciones de la herida & $19(6.1)$ \\
\hline Mortalidad & $18(5.8)$ \\
\hline \multicolumn{2}{|l|}{ Comorbilidad } \\
\hline Diabetes mellitus (DM) & $21(6.8)$ \\
\hline Hipertensión arterial (HAS) & $37(11.9)$ \\
\hline DM e HAS & $36(11.6)$ \\
\hline Otra & $12(3.8)$ \\
\hline \multicolumn{2}{|l|}{ Cirugía realizada } \\
\hline Disección radical tipo I & $3(1)$ \\
\hline Disección radical modificada tipo III & $78(25)$ \\
\hline Disección suprahomohiodea & $48(15.4)$ \\
\hline Disección lateral & $108(34.7)$ \\
\hline Disección posterolateral & $29(9.3)$ \\
\hline Disección anterior & $25(8)$ \\
\hline Disección lateral y anterior & $19(6.1)$ \\
\hline Disección posterior & $1(0.3)$ \\
\hline \multicolumn{2}{|l|}{ Resultado histopatológico } \\
\hline Positivo & $264(84.9)$ \\
\hline Negativo & $47(15.1)$ \\
\hline
\end{tabular}

el sexo masculino (OR: 0.2; IC 95\%: 0.7-0.8; $p=0.02$ ) y la disección radical de tipo III (OR: 3.1; IC 95\%: $0.9-10 ; p=0.05$ ).

\section{Mortalidad}

Fallecieron 18 pacientes (tasa de mortalidad del $5.8 \%$ ), de los cuales ocho a causa de hemorragia grave posquirúrgica, cinco por sepsis, dos por complicaciones asociadas a una fístula quilosa y el resto por complicaciones no relacionadas con el procedimiento. Los factores que se asociaron con la 
Tabla 2. Factores asociados a morbilidad (complicaciones)

\begin{tabular}{|c|c|c|c|c|c|c|c|c|}
\hline \multirow[t]{2}{*}{ Variable } & \multicolumn{2}{|c|}{ Vascular } & \multicolumn{2}{|c|}{ Nerviosa } & \multicolumn{2}{|c|}{ Herida } & \multicolumn{2}{|c|}{ Fístula quilosa } \\
\hline & Sí/No & $\mathbf{p}^{*}$ & Sí/No & $p^{*}$ & Sí/No & $p^{*}$ & Sí/No & $p^{*}$ \\
\hline \multicolumn{9}{|l|}{ Sexo } \\
\hline Masculino & $11 / 108$ & 0.12 & $7 / 108$ & 0.04 & $12 / 108$ & 0.009 & $8 / 108$ & 0.02 \\
\hline Femenino & $12 / 203$ & & $4 / 203$ & & 7/203 & & 4/203 & \\
\hline \multicolumn{9}{|l|}{ Comorbilidad } \\
\hline Diabetes mellitus & $6 / 57$ & 0.22 & $4 / 57$ & 0.12 & $7 / 57$ & 0.04 & $2 / 57$ & 0.61 \\
\hline Hipertensión arterial & $10 / 73$ & 0.02 & $1 / 73$ & 0.22 & $7 / 73$ & 0.12 & $3 / 73$ & 0.56 \\
\hline \multicolumn{9}{|l|}{ Nivel clínico } \\
\hline I & $13 / 129$ & 0.09 & $10 / 129$ & 0.001 & $12 / 129$ & 0.04 & $7 / 129$ & 0.18 \\
\hline$\|$ & $22 / 272$ & 0.18 & $11 / 272$ & 0.22 & $18 / 272$ & 0.28 & $11 / 272$ & 0.54 \\
\hline III & $22 / 276$ & 0.24 & $11 / 276$ & 0.26 & $19 / 276$ & 0.09 & $11 / 276$ & 0.60 \\
\hline IV & 9/217 & 0.12 & $3 / 217$ & 0.004 & $15 / 217$ & 0.26 & $11 / 217$ & 0.07 \\
\hline V & $12 / 87$ & 0.009 & $3 / 87$ & 0.63 & 8/87 & 0.12 & $7 / 87$ & 0.02 \\
\hline VI & $3 / 58$ & 0.34 & $0 / 58$ & 0.09 & $2 / 58$ & 0.27 & $2 / 58$ & 0.60 \\
\hline VII & $3 / 46$ & 0.54 & $0 / 46$ & 0.16 & $1 / 46$ & 0.19 & $2 / 46$ & 0.55 \\
\hline \multicolumn{9}{|l|}{ Nivel quirúrgico } \\
\hline l & 3/66 & 0.23 & 9/66 & 0.00 & 6/66 & 0.19 & $1 / 66$ & 0.23 \\
\hline$\|$ & $14 / 177$ & 0.43 & $11 / 177$ & 0.002 & $14 / 177$ & 0.09 & 7/177 & 0.58 \\
\hline III & $19 / 185$ & 0.01 & $6 / 185$ & 0.48 & $13 / 185$ & 0.28 & $10 / 185$ & 0.07 \\
\hline IV & $9 / 100$ & 0.29 & $1 / 100$ & 0.08 & $5 / 100$ & 0.38 & $6 / 100$ & 0.015 \\
\hline V & $3 / 28$ & 0.34 & $0 / 28$ & 0.34 & $2 / 28$ & 0.52 & $2 / 28$ & 0.29 \\
\hline VI & $2 / 45$ & 0.32 & $0 / 45$ & 0.17 & $2 / 45$ & 0.46 & $0 / 45$ & 0.14 \\
\hline VII & $1 / 16$ & 0.66 & $0 / 16$ & 0.55 & $0 / 16$ & 0.35 & $0 / 16$ & 0.52 \\
\hline Sintomatología & $16 / 145$ & 0.019 & $8 / 145$ & 0.07 & $14 / 145$ & 0.01 & $10 / 145$ & 0.009 \\
\hline \multicolumn{9}{|l|}{ Tipo de cirugía } \\
\hline Disección radical tipo III & $11 / 78$ & 0.01 & $4 / 78$ & 0.28 & $8 / 78$ & 0.07 & $6 / 78$ & 0.05 \\
\hline Disección suprahomohiodea & $0 / 48$ & 0.01 & $7 / 48$ & 0.00 & $3 / 48$ & 0.58 & $0 / 48$ & 0.12 \\
\hline Disección lateral & $8 / 108$ & 0.58 & 0/108 & 0.008 & $3 / 108$ & 0.05 & $4 / 108$ & 0.59 \\
\hline Disección posterolateral & $0 / 29$ & 0.09 & $0 / 29$ & 0.33 & $2 / 29$ & 0.54 & $0 / 29$ & 0.30 \\
\hline Disección anterior & $0 / 25$ & 0.13 & $0 / 25$ & 0.39 & $0 / 25$ & 0.19 & $0 / 25$ & 0.35 \\
\hline Disección lateral y anterior & $3 / 19$ & 0.15 & $0 / 19$ & 0.49 & 2/19 & 0.32 & $1 / 19$ & 0.53 \\
\hline
\end{tabular}

*Mediante prueba de ji al cuadrado o prueba exacta de Fisher.

mortalidad fueron la presencia de síntomas clínicos (OR: 4.32; IC 95\%: 1.3-13.4; $p=0.006$ ), las metástasis en el nivel I (OR: 4.41; IC 95\%: 1.5-10.9; $p=0.005$ ), la disección radical modificada de tipo 3 (OR: 3.2; IC $95 \%: 1.2-8.5 ; p=0.01$ ) y las complicaciones vasculares (OR: 11; IC 95\%: 3.7-32.2; $p=0.0001$ ) y de la herida (OR: 5.29; IC 95\%: 1.5-18.0; $p=0.01$ ), como se observa en la tabla 3 .

\section{Discusión}

La linfadenectomía cervical ganglionar en pacientes con tumores de cabeza y cuello continúa siendo considerada como la primera línea de tratamiento para las metástasis linfáticas de los tumores de esta región, así como una de las principales herramientas para la estadificación al considerarse como el factor pronóstico más importante de malignidad de las neoplasias de las vías aerodigestivas ${ }^{1,2}$.
Como se pudo observar en nuestro estudio, existe un gran número de tumores que originan metástasis en los ganglios linfáticos cervicales, dentro de los cuales encontramos que la gran mayoría corresponden a tumores de origen tiroideo, siendo estos el $62 \%$ en nuestra población, mientras que en la literatura mundial podemos encontrar porcentajes de hasta el $72 \%$, que se asocian con una baja letalidad pero con una alta incidencia ${ }^{3}$. Si englobamos los tumores de las vías aerodigestivas en general, encontramos una incidencia de metástasis cervicales alrededor del $16 \%$, más alta si se compara con la literatura, en la que es del 12\%; sin embargo, en contraste con los resultados obtenidos en estudios realizados en otros países, observamos que las neoplasias de la laringe en nuestro estudio ocupan el segundo lugar ${ }^{6,10,11}$.

De acuerdo con la clasificación propuesta desde 1987 por la American Head and Neck Society (AHNS) y la American Academy of Otolaryngology Head and 
Tabla 3. Análisis univariado de factores asociados a la mortalidad en la población de estudio

\begin{tabular}{|c|c|c|c|}
\hline Variable & $\mathrm{n}=18$ & $p^{*}$ & OR (IC 95\%) \\
\hline \multicolumn{4}{|l|}{ Sexo } \\
\hline Masculino & $9 / 108$ & 0.12 & $0.5(0.1-1.3)$ \\
\hline Femenino & $9 / 203$ & & \\
\hline \multicolumn{4}{|l|}{ Diagnóstico } \\
\hline Cáncer de tiroides & $9 / 194$ & 0.19 & $0.58(0.2-1.5)$ \\
\hline Melanoma & $2 / 11$ & 0.12 & $3.94(0.7-19.7)$ \\
\hline Cáncer de laringe & $2 / 22$ & 0.36 & $1.7(0.3-7.9)$ \\
\hline Cáncer epidermoide & $1 / 5$ & 0.25 & $4.2(0.4-40.1)$ \\
\hline \multicolumn{4}{|l|}{ Cirugía realizada } \\
\hline Disección radical tipo III & $9 / 78$ & 0.01 & $3.24(1.2-8.5)$ \\
\hline Disección suprahomohiodea & $3 / 48$ & 0.54 & $1.10(0.3-3.9)$ \\
\hline Disección lateral & $4 / 108$ & 0.18 & $0.51(0.16-1.6)$ \\
\hline Disección anterior & $1 / 25$ & 0.56 & $0.65(0.08-5.1)$ \\
\hline Disección posterolateral & $0 / 29$ & 0.16 & $0.93(0.9-0.9)$ \\
\hline Disección lateral y anterior & $1 / 19$ & 0.69 & $0.89(0.1-7.1)$ \\
\hline \multicolumn{4}{|l|}{ Complicaciones } \\
\hline Vasculares & $7 / 23$ & 0.0001 & $11(3.7-32.2)$ \\
\hline Nerviosas & $1 / 11$ & 0.48 & $1.66(0.2-13.7)$ \\
\hline Herida & $4 / 19$ & 0.01 & $5.29(1.5-18.0)$ \\
\hline Fístula quilosa & $2 / 12$ & 0.14 & $3.53(0.7-17.5)$ \\
\hline Resultado de patología & 17 & 0.21 & $3.16(0.4-24.3)$ \\
\hline Sintomatología presente & 14 & 0.006 & $4.32(1.3-13.4)$ \\
\hline \multicolumn{4}{|l|}{ Nivel ganglionar } \\
\hline I & 9 & 0.005 & $4.41(1.5-10.9)$ \\
\hline$\|$ & 12 & 0.27 & $1.52(0.5 .4 .2)$ \\
\hline III & 14 & 0.81 & $2.94(0.8-7.7)$ \\
\hline IV & 6 & 0.54 & $1.05(0.3-2.9)$ \\
\hline V & 3 & 0.21 & $2.14(0.5-7.9)$ \\
\hline $\mathrm{VI}$ & 2 & 0.50 & $0.72(0.1-3.2)$ \\
\hline VII & 0 & 0.61 & $0.94(0.9-0.9)$ \\
\hline
\end{tabular}

${ }^{*}$ Mediante prueba de ji al cuadrado o prueba exacta de Fisher.

IC 95\%: intervalo de confianza del 95\%; OR: odds ratio.

Neck Surgery (AAOHNS), se pudo apreciar que los niveles más afectados son el II, el III y el IV, el cual, si se analiza el drenaje linfático propio de cada tumor, corresponde a la evolución natural de su diseminación ${ }^{7,12,13}$, ya que tienen un patrón predecible y secuencial, como se observa en los tumores de tiroides, lengua, encía, laringe y parótida, que tienen drenaje hacia estos niveles y por lo tanto existe correlación con los niveles ganglionares resecados, con una menor proporción de los niveles V, VI y VII. Al analizar los pacientes asintomáticos en comparación con los que presentan nódulos o masas cervicales, vemos que estos son un parámetro pronóstico para la recurrencia, ya que todos los que cursan con sintomatología resultan positivos a malignidad en el estudio histopatológico, siendo estadísticamente significativos, lo que concuerda con los hallazgos de Sugitani, et al. $^{13}$, quienes encontraron que el $20.6 \%$ de los pacientes con ganglios linfáticos palpables desarrollaron recurrencia nodal, considerando de tal forma como pacientes de alto riesgo aquellos que presentaban ganglios linfáticos de $3 \mathrm{~cm}$ o más.

De acuerdo con la evolución quirúrgica de las linfadenectomías, cada vez se opta más por preferir un manejo conservador, al evidenciarse en estudios previos que la resección de estructuras no ganglionares, como el nervio espinal, músculos o vasos sanguíneos, no ofrece ventaja en la sobrevida global ni en el periodo libre de enfermedad ${ }^{14-16}$. Coincidimos con la literatura mundial al realizar cirugías más conservadoras, con resección de niveles ganglionares por grupos dependiendo de los niveles afectados y la posibilidad de metástasis de manera contigua, por lo cual la disección lateral de los niveles II, III y IV continúa siendo la cirugía más realizada, seguida por la disección radical modificada de tipo 3 que incluye los niveles ganglionares I a $\mathrm{V}$, conservando estructuras no ganglionares, o la disección suprahomohioidea para niveles superiores. La extensión de la linfadenectomía influye directamente en la morbilidad, principalmente con afecciones vasculares, al realizar disecciones extensas como la disección radical modificada.

La presencia de nódulos en niveles yugulares y su resección afectan de manera significativa el aumento de hematomas y de perforaciones incidentales en la vena yugular, que en correlación con la extensión de la linfadenectomía en una disección radical puede presentarse hasta en un $4 \%$ según la literatura ${ }^{6,11}$ (un (5\% en nuestro estudio).

La incidencia de complicaciones quirúrgicas es variable, dependiente de cada cirujano y de cada hospital, además de correlacionarse con el tipo histológico del tumor ${ }^{17}$. En nuestro estudio encontramos una tasa de complicaciones del $17 \%$, dentro de las que se incluyen las nerviosas, las vasculares y las propias de la herida, en contraste con lo reportado por Dedivitis, et al. ${ }^{18}$, quienes encontraron una frecuencia de hasta el $28 \%$. Las complicaciones más frecuente en nuestra población fueron las vasculares, a diferencia del estudio previamente mencionado ${ }^{18}$, en el que las complicaciones nerviosas (daño al ramo marginal del nervio mandibular, nervio accesorio) fueron las más comunes.

Para las complicaciones nerviosas se ha reportado una incidencia, según la literatura internacional, del $1-2 \%{ }^{19}$; sin embargo, no se cuenta con información a largo plazo de los posibles beneficios de la 
rehabilitación, en contraste con las evidencias ${ }^{11,12}$ que revelan una mejoría en un gran porcentaje de pacientes, con solo un $3 \%$ que permanecerán con un déficit permanente sensorial o motor. En nuestro estudio no encontramos lesiones del nervio espinal, mientras que sí las encontramos del nervio hipogloso mayor o del ramo marginal del facial (3.5\%), presentando como resultado debilidad lingual homolateral, desviación de la lengua hacia el lado afectado y, en menor proporción, dificultad con el habla y la deglución (solo un paciente presentó estos déficits).

Las complicaciones propias de la herida tuvieron una frecuencia del $6.1 \%$, menor que lo observado en la serie de Pellini, et al. ${ }^{20}$, en la que informan hasta un $20 \%$. En mayor proporción se observaron dehiscencia de la herida e infección, y se encontraron como factores asociados la presencia de diabetes mellitus y el haber recibido radioterapia externa; y son directamente proporcionales a las tasas de mortalidad observadas en este estudio. Por ello, el manejo con antimicrobianos profilácticos, así como el manejo gentil de los tejidos irradiados, de acuerdo con lo informado en la literatura, reducen la incidencia de infecciones de la herida.

De acuerdo con lo informado en la literatura internacional ${ }^{8,10,17}$, la presencia de fístula quilosa se observa en el $1-2.5 \%$ de los casos. En nuestro estudio las observamos en el $1.9 \%$ y se resolvieron con manejo médico; un paciente requirió cirugía y no se demostró impacto directo en la mortalidad.

La linfadenectomía cervical es la primera opción de tratamiento en pacientes con metástasis ganglionares cervicales de neoplasias de la cabeza y del cuello20, y también con neoplasias distantes que dan origen a metástasis en el cuello, como tumores de pulmón, mama, endometrio y sarcomas. Su manejo se considera seguro, con un porcentaje de complicaciones menor del $7 \%$. Aún seguimos observando pacientes con tumores con pronóstico desfavorable, de grandes dimensiones, que ulceran e infiltran la piel, siendo estos los que tienen la mayor tasa de complicaciones, como dehiscencias de herida y afecciones vasculares; la disminución de su incidencia solo podrá lograrse con técnicas quirúrgicas cuidadosas e indicaciones terapéuticas claras ${ }^{21,22}$.

\section{Conclusiones}

La linfadenectomía cervical es el tratamiento idóneo para el control regional en pacientes con metástasis ganglionares, evitando la resección innecesaria de estructuras no linfáticas. Se observó una morbilidad general del $17 \%$, semejante a la registrada en otros centros especializados. Se ha optado por realizar cirugías más conservadoras, siempre y cuando se cumpla con el control regional de la enfermedad, dada la relación directa entre las metástasis linfáticas y el incremento tanto en la persistencia como en la recurrencia neoplásica. Debido a que la linfadenectomía cervical constituye un elemento para estadificar las neoplasias originadas en la cabeza y el cuello, y en aquellas que son metástasis de neoplasias a distancia, se destaca la importancia de su realización ya que el beneficio de la resección ganglionar sobrepasa la baja tasa de complicaciones esperadas de dicha cirugía.

\section{Bibliografía}

1. Cadena E, Sanabria A. Disección ganglionar de cuello: conceptos actuales. Rev Colomb Cancerol. 2011;15:31-40.

2. Pellini R, Mercante G, Marchese C, Terenzi V, Sperduti I, Manciocco V, et al. Predictive factors for postoperative wound complications after neck dissection. Spriano Acta Otorhinolaryngologica Italica. 2013;33:16-22.

3. Chan JY. Efficacy of neck dissection in the management of isolated nodal recurrence after head and neck cancer treatment. Curr Oncol Rep. 2013;15:142-5.

4. Alfio Ferlito K, Robbins T, Silver CE, Hasegawa Y, Rinaldo A. Classification of neck dissections: an evolving system. Auris Nasus Larynx. 2009;36:127-34.

5. Chummun S, McLean NR, Ragbir M. Surgical education: neck dissection. Br J Plast Surg. 2004:57:610-23.

6. Prim MP, De Diego JI, Verdaguer JM, Sastre N, Rabanl I. Neurologic complications following neck dissection. Eur Arch Otorhinolaryngol. 2006;263:473-6.

7. Ducic Y, Young L, McIntyre J. Neckissection: Past and present. Minerva Chir. 2010;65(1):45-58.

8. Smoke A, Delegge MH. Chyle leaks: consensus on management? Nutr Clin Pract. 2008:23:529-32.

9. Pellini R, Mercante G, Marchese C, Terenzi V, Sperduti I, Manciocco V, et al. Predictive factors for postoperative wound complications after neck dissection. Acta Otorhinolaryngol. 2013;33:16-22.

10. Ferlito A, Rinaldo A, Robbins KT, Silver CE. Neck dissection: past, present and future? J Laryngol Otol. 2006;120:87-92.

11. Ferlito A, Rinado A, Silver CE, Shah JP, Suárez C, Medina JE, et al. Neck dissection: then and now. Auris Nasus Larynx. 2006;33(4):365-74.

12. Ferlito A, Robbins KT, Shah JP, Medina JE, Silver CE, Al-Tamimi S, et al. Proposal for a rational classification of neck dissections. Head Neck. 2011;33:445-50.

13. Sugitani I, Fujimoto Y, Yamada K, Yamamoto N. Prospective outcomes of selective lymph node dissection for papillary thyroid carcinoma based on preoperative ultrasonography. World J Surg. 2008;32:2494-502.

14. Nobuyuki W, Quan-Yang D, Kiminori S, Hiroyuki I, Kaori K, Takashi M, et al. Lymph node metastasis from 259 papillary thyroid microcarcinomas. Frequency, pattern of occurrence and recurrence, and optimal strategy for neck dissection. Ann Surg. 2003;237:399-407.

15. Morestin $\mathrm{H}$. The evidence of cervical nodes in cancers of the mouth and pharynx. J Chir. 1993;10:657-93.

16. Rigual NR, Wiseman SM. Neck dissection: current concepts and future directions. Surg Oncol Clin Am. 2004;13:151-66.

17. Giugliano G, Proh M, Gibelli B, Grosso E, Tagliabue M, De Fiori E, et al. Central neck dissection in differentiated thyroid cancer: technical notes. Acta Otorhinolaryngol Ital. 2014;34:9-14.

18. Dedivitis RA, Guimarães AV, Pfuetzenreiter EG Jr, Castro MA. Neck dissection complications. Braz J Otorhinolaryngol. 2011;77:65-9.

19. Hughes DT, Doherty GM. Central neck dissection for papillary thyroid cancer. Cancer Control. 2011;18:83-8.

20. Pellini R, Mercante G, Marchese C, Terenzi V, Sperduti I, Manciocco V, et al. Predictive factors for postoperative wound complications after neck dissection. Acta Otorhinolaryngol Ital. 2013;33:16-22.

21. Shoppy WD, Holsinger FC. Management of the neck in thyroid cancer. Otolaryngol Clin N Am. 2014;47:545-56.

22. Kerawala CJ. Complications of head and neck cancer surgery. Prevention and management. Oral Oncology. 2010;46:433-5. 